\title{
Therapeutic effects of stiripentol against ischemia-reperfusion injury in gerbils focusing on cognitive deficit, neuronal death, astrocyte damage and blood brain barrier leakage in the hippocampus
}

\author{
Myoung Cheol Shin ${ }^{1, \#}$, Tae-Kyeong Lee ${ }^{2, \#,}$, Jae-Chul Lee ${ }^{3}$, Hyung II Kim ${ }^{1,4}$, Chan Woo Park', Jun Hwi Cho', \\ Dae Won Kim ${ }^{5}$, Ji Hyeon Ahn ${ }^{3,6}$, Moo-Ho Won ${ }^{3, *}$, and Choong-Hyun Lee ${ }^{7, *}$
}

\begin{abstract}
'Department of Emergency Medicine, Kangwon National University Hospital, Kangwon National University School of Medicine, Chuncheon $24289,{ }^{2}$ Department of Biomedical Science, Research Institute of Bioscience and Biotechnology, Hallym University, Chuncheon 24252, ${ }^{3}$ Department of Neurobiology, Kangwon National University School of Medicine, Chuncheon 24341, ${ }^{4}$ Department of Emergency Medicine, Dankook University Hospital, Dankook University College of Medicine, Cheonan 31116, ${ }^{5}$ Department of Biochemistry and Molecular Biology, Research Institute of Oral Sciences, College of Dentistry, GangnungWonju National University, Gangneung 25457, ${ }^{6}$ Department of Physical Therapy, College of Health Science, Youngsan University, Yangsan 50510, ${ }^{7}$ Department of Pharmacy, College of Pharmacy, Dankook University, Cheonan 31116, Korea
\end{abstract}

\section{ARTICLE INFO \\ Received October 25, 2021 \\ Revised November 23, 2021 \\ Accepted November 30, 2021 \\ *Correspondence \\ Moo-Ho Won \\ E-mail:mhwon@kangwon.ac.kr \\ Choong-Hyun Lee \\ E-mail:anaphy@dankook.ac.kr}

\section{Key Words}

Blood-brain barrier

Brain ischemia

Hippocampus

Neuroprotection

Stiripentol

\#These authors contributed equally to this work.
ABSTRACT Stiripentol is an anti-epileptic drug for the treating of refractory status epilepticus. It has been reported that stiripentol can attenuate seizure severity and reduce seizure-induced neuronal damage in animal models of epilepsy. The objective of the present study was to investigate effects of post-treatment with stiripentol on cognitive deficit and neuronal damage in the cornu ammonis 1 (CA1) region of the hippocampus proper following transient ischemia in the forebrain of gerbils. To evaluate ischemia-induced cognitive impairments, passive avoidance test and 8-arm radial maze test were performed. It was found that post-treatment with stiripentol at $20 \mathrm{mg} / \mathrm{kg}$, but not 10 or $15 \mathrm{mg} / \mathrm{kg}$, reduced ischemia-induced memory impairment. Transient ischemia-induced neuronal death in the CA1 region was also significantly attenuated only by $20 \mathrm{mg} / \mathrm{kg}$ stiripentol treatment after transient ischemia. In addition, $20 \mathrm{mg} / \mathrm{kg}$ stiripentol treatment significantly decreased ischemia-induced astrocyte damage and immunoglobulin G leakage. In brief, stiripentol treatment after transient ischemia ameliorated transient ischemia-induced cognitive impairment in gerbils, showing that pyramidal neurons were protected and astrocyte damage and blood brain barrier leakage were significantly attenuated in the hippocampus. Results of this study suggest stiripentol can be developed as a candidate of therapeutic drug for ischemic stroke.

\section{INTRODUCTION}

Transient ischemia in the brain can cause metabolic and neurochemical alterations and lead to selective neuronal loss (death) in vulnerable regions after a transient ischemia $[1,2]$. In particular, neuronal loss occurs in the cornu ammonis (CA) 1 field in the hippocampus proper consisting of CA1 to CA3 fields at a few (four to five) days after five minutes of transient forebrain ischemia (TFI), which is called "delayed neuronal death" [3]. Although the exact mechanism of TFI-induced delayed neuronal death in the hippocampal CA1 field has not been clearly elucidated yet, many previous studies have suggested some underlying mechanisms (i) $\$$ This is an Open Access article distributed under the terms of the Creative Commons Attribution Non-Commercial License, which permits unrestricted non-commercial use, distribution, and reproduction in any medium, provided the original work is properly cited. Copyright @ Korean J Physiol Pharmacol, pISSN 1226-4512, eISSN 2093-3827
Author contributions: M.H.W. and C.H.L. conception and design of study; H.I.K., C.W.P., and D.W.K. acquisition of data; J.C.L., J.H.C., and D.W.K. analysis and/or interpretation of data; M.C.S. and T.K.L. drafting the manuscript; M.H.W. revising the manuscript critically for important intellectual content. 
such as excitotoxicity and oxidative stress [4-6]. Neuroinflammatory processes including glial activation and enhanced production and release of inflammatory cytokines have also been thought as major mechanisms $[2,7,8]$. In addition to delayed neuronal death, it has been demonstrated that TFI can cause the damage of bloodbrain barrier (BBB) integrity, leading to leakage and increased permeability (i.e., vasogenic edema, abnormal exchange of molecules, etc.) [9-12].

Stiripentol (STP) is a structurally unique anti-epileptic drug for treating Dravet syndrome and refractory status epilepticus [13]. Previous studies have suggested some underlying mechanisms of the anticonvulsant effect of STP such as action on gammaaminobutyric acid A receptor and inhibition of lactate dehydrogenase $[14,15]$. In addition, it has been reported that STP can reduce seizure severity and seizure-induced neuronal damage in animal models of epilepsy [16-18]. In addition, a recent study has reported the effect of STP against neuropathic pain induced by spinal nerve transection in mice, showing that intrathecal administration with STP can attenuate mechanical hyperalgesia in a dose-dependent manner [19].

Although anti-epileptic and neuroprotective effects of STP have been demonstrated in epileptic seizure models, studies investigating whether STP has protective effect against ischemic damage induced by TFI have not been reported yet. Therefore, the objective of this study was to investigate the effects of post-treatment with STP after TFI on TFI-induced cognitive impairment, neuronal damage/death, astrocyte damage and BBB leakage in the hippocampal CA1 region using the gerbil as a good model of TFI $[2,20,21]$.

\section{METHODS}

\section{Experimental animals}

Male Mongolian gerbils (total $\mathrm{n}=144 ; 80 \pm 5 \mathrm{~g}$ of body weight; six months of age) were obtained from Experimental Animal Center of Kangwon National University (Chuncheon, Korea). They were housed under optimal conditions $\left(24 \pm 0.5^{\circ} \mathrm{C}\right.$ of room temperature; $55 \pm 5 \%$ of relative humidity; $12: 12 \mathrm{~h}$ of light/dark cycle) with three to five animals per cage. Freely accessible pellet feed (DBL Co., Ltd., Eumseong, Korea) and potable water were provided to the gerbils. Entrance to the room was rigorously regulated to reduce superfluous stress to the gerbils.

In this experiment, the experimental protocol was approved (approval no., KW-200113-1) by "Institutional Animal Care and Use Committee" of Kangwon National University on February 18,2020 .

\section{Experimental groups}

In this study, experimental groups were as follows: 1) Sham- vehicle group $(\mathrm{n}=15)$ was given sham operation and treated with vehicle (saline); 2) TFI-vehicle group ( $\mathrm{n}=21$ ) was given TFI operation and treated with vehicle; 3), 4), and 5) Sham-10, 15, and 20 $\mathrm{mg} / \mathrm{kg}$ STP groups ( $\mathrm{n}=15$, respectively) were given sham operation and treated with 10,15 , and $20 \mathrm{mg} / \mathrm{kg}$ STP, respectively; 6), 7), and 8) TFI-10, 15, and $20 \mathrm{mg} / \mathrm{kg}$ STP groups ( $\mathrm{n}=21$, respectively) were given TFI operation and treated with 10,15 , and $20 \mathrm{mg} / \mathrm{kg}$ STP, respectively.

\section{Surgery for TFI and administration of STP}

TFI was induced in accordance with a previously published method $[4,12]$. In brief, the gerbils were anesthetized by inhaling 2.5\% isoflurane (Hana Pharm. Co., Ltd., Seoul, Korea) in $33 \% \mathrm{O}_{2}$ and $67 \% \mathrm{~N}_{2} \mathrm{O}$. Under anesthesia, the ventral necks of the gerbils were shaved, and a midline incision $(1.2 \pm 0.2 \mathrm{~cm})$ was made. Next, bilateral common carotid arteries (BCCA) were isolated and ligated with aneurysm clips $(0.69 \mathrm{~N})$ (Yasargil FE 723K; Aesculap, Tuttlingen, Germany). To verify complete occlusion of the BCCA, the central arteries located in bilateral retinae were observed using an ophthalmoscope (HEINE K180) (Heine Optotechnik, Herrsching, Germany). After the conformation of perfect ischemia, the clips were removed at 5 min after BCCA ligation, and the incision was closed using 3-0 suture silk (Ethicon Inc., Somerville, NJ, USA). During the surgical procedure, body temperature was monitored in real-time with a rectal temperature probe (TR-100) (Fine Science Tools, Foster City, CA, USA), and body temperature was controlled at normothermic condition (37 $\pm 0.5^{\circ} \mathrm{C}$ ). In this experiment, the sham operation was performed like TFI procedure without BCCA ligation.

For the administration of STP (synonyms, 4,4-Dimethyl1-[(3,4-methylenedioxy)phenyl]-1-penten-3-ol and Diacomit; molecular formula, $\mathrm{C}_{14} \mathrm{H}_{18} \mathrm{O}_{3}$; solubility, DMSO: $\geq 20 \mathrm{mg} / \mathrm{ml}$ ), it was dissolved in vehicle (saline) in order that the final dosage was to be $10 \mathrm{mg}, 15 \mathrm{mg}$, and $20 \mathrm{mg}$ per $1 \mathrm{~kg}$ of body weight. The gerbils were intraperitoneally injected with vehicle or STP at $30 \mathrm{~min}$ after sham or TFI operation (Fig. 1).

\section{Behavioral tests}

\section{Passive avoidance test (PAT)}

To evaluate short term memory following TFI, PAT was conducted in the light of previous studies [12,22]. In short, we used the Gemini Avoidance System (GEM 392) (San Diego Instruments, San Diego, CA, USA) which consists of dark and light sections communicating by a vertical sliding door between the two sections. As shown in Fig. 1, PAT was performed by two phases at one day before TFI and five days after TFL. Namely, training was performed, and substantive trial was conducted at $20 \mathrm{~min}$ after the training. In the training, the gerbil was placed in the light section and allowed to freely explore both sections for one minute while the vertical door was opened. When the gerbil entered the 


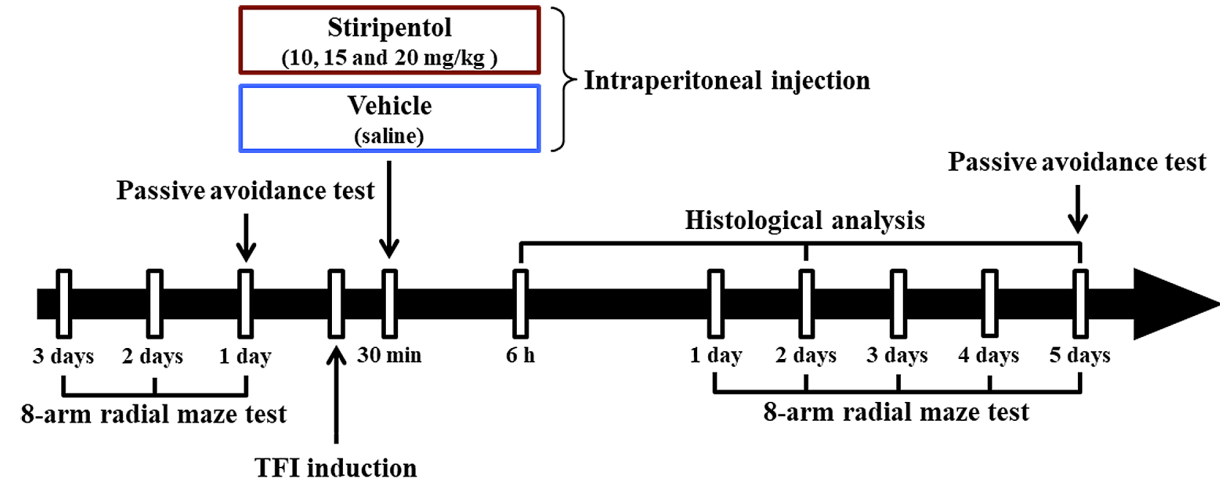

Fig. 1. Experimental timeline. Vehicle (saline) and STP (10, 15, and $20 \mathrm{mg} / \mathrm{kg}$ ) are treated $30 \mathrm{~min}$ after TFI. 8-ARMT is conducted at 3,2 , and 1 day before TFI, and $1,2,3,4$, and 5 days after TFI. PAT is carried out at 1 day before, and 5 days after TFI. The animals are sacrificed at 6 h, 2 and 5 days after TFI respectively, and histologically analyzed. STP, stiripentol; TFI, transient forebrain ischemia; 8-ARMT, 8-arm radial maze test; PAT, passive avoidance test.

Table 1. List of primary and secondary antibodies used for immunohistochemistry

\begin{tabular}{lll}
\hline \multicolumn{1}{c}{ Antibody } & Dilute rates & \multicolumn{1}{c}{ Manufacturers } \\
\hline Primary antibodies & & \\
$\quad$ Mouse anti-neuronal nuclei (NeuN) & $1: 1,000$ & Chemicon, Temecula, CA, USA \\
Rabbit anti-ionized calcium-binding adapter molecule 1 (Iba-1) & $1: 800$ & Wako, Osaka, Japan \\
$\quad$ Mouse anti-glial fibrillary acidic protein (GFAP) & $1: 1,000$ & Chemicon, Temecula, CA, USA \\
$\quad$ Rabbit anti-Mongolian gerbil immunoglobulin G (IgG) & $1: 1,000$ & Bioss antibodies, Atlanta, GA, USA \\
Secondary antibodies & & \\
$\quad$ Biotinylated horse anti-mouse IgG & $1: 250$ & Vector Laboratories Inc., Burlingame, CA, USA \\
Biotinylated goat anti-rabbit IgG & $1: 250$ & Vector Laboratories Inc., Burlingame, CA, USA \\
\hline
\end{tabular}

dark section, the door was closed and an electric foot-shock $(0.5$ $\mathrm{mA}$ for $5 \mathrm{sec}$ ) was given to the gerbil from steel grid on the floor. For the substantive trial, the gerbil was placed in the light section, and the latency time to enter the dark section was recorded within three minutes.

\section{8-arm radial maze test (8-ARMT)}

8-ARMT was performed in order to evaluate spatial memory following TFI in accordance with previously described methods $[4,11,12]$. Briefly, a central platform consisting of a nontransparent acryl board (diameter, $20 \mathrm{~cm}$ ) with radially extended eight arms (width, $5 \mathrm{~cm}$; height, $9 \mathrm{~cm}$; length, $35 \mathrm{~cm}$ ) (Stoelting Co., Wood Dale, IL, USA) was used. Pelleted diets (DBL Co., Ltd.) were located at the end of each arm. As shown in Fig. 1, the gerbils were pre-trained once a day for three days before TFI induction. The substantive trials were conducted once a day for five days from one day after TFI induction. To evaluate 8-ARMT, the gerbil was placed onto the central platform, and the numbers of errors were recorded at every occasion when the gerbil went into the arm that they had already entered before. When the gerbil ate up the pellets, each trial was terminated.

\section{Preparation of histological sections}

As shown in Fig. 1, the gerbils used for all groups were sacrificed ( $n=5$, respectively, in the sham groups; $n=7$, respectively, in the TFI groups) at six hours, two days and five days after sham or TFI operation. According to our previous studies [4,12], brain tissue sections containing the hippocampus were prepared. In short, the gerbils were profoundly anesthetized by intraperitoneal administration of $90 \mathrm{mg} / \mathrm{kg}$ pentobarbital sodium (JW pharm. Co., Ltd., Seoul, Korea). Under deep anesthesia, the brains of the gerbils were rinsed with saline through ascending aorta and fixed 4\% paraformaldehyde (in $0.1 \mathrm{M}$ phosphate buffer; $\mathrm{pH}$ 7.4). The brains were harvested and more fixed with same fixative for four hours at room temperature. To cryoprotect the brains when they were sectioned, the fixed brains were infiltrated with $25 \%$ sucrose solution (in $0.1 \mathrm{M} \mathrm{PB}$; $\mathrm{pH} 7.4$ ) for $24 \mathrm{~h}$ at room temperature. Using a sliding microtome (SM2020 R) (Leica, Nussloch, Germany) equipped with BFS-40MP freezing platform (Physitemp Instruments, Inc., Clifton, NJ, USA), the brains containing the hippocampus were coronally sectioned into $30 \mu \mathrm{m}$ of thickness. The sections used in this experiment were selected at level of $-1.8 \mathrm{~mm}$ to $2.7 \mathrm{~mm}$ of antero-posterior to the bregma in the light of the gerbil brain atlas [23].

\section{Immunohistochemistry}

Immunohistochemical studies were done to examine changes in neurons, astrocytes, and immunoglobulin $\mathrm{G}(\mathrm{IgG})$ using the following primary antibodies: neuronal nuclei (NeuN; a marker for neurons), and IgG (Table 1). In brief, in accordance with previously published methods $[4,12]$, the sections were incubated in $0.3 \% \mathrm{H}_{2} \mathrm{O}_{2}$ for $25 \mathrm{~min}$ at room temperature, and they were immersed in five percent normal horse or goat serum for $30 \mathrm{~min}$ at room temperature. Thereafter the sections were immunoreacted with each primary antibody for $24 \mathrm{~h}$ at $4^{\circ} \mathrm{C}$. After washing them, 
the immunoreacted sections were reacted with corresponding secondary antibodies for two hours at room temperature followed by incubated in diluted avidin-biotin complex (dilute rate, 1:250) (Vector Laboratories, Burlingame, CA, USA) (Table 1). After washing, these sections were subsequently visualized via immersing into 0.06\% 3,3'-diaminobenzidine tetrahydrochloride (SigmaAldrich Co., St. Louis, MO, USA) containing $0.003 \% \mathrm{H}_{2} \mathrm{O}_{2}$. Finally, these sections were mounted onto the slide glass, dehydrated using ethyl alcohol series, cleared in xylene, and coverslipped with Canada balsam (Kanto Chemical, Tokyo, Japan).

To evaluate the change of NeuN immunoreactive cells (neurons) was performed according to published papers $[4,24]$ with minor modification. The image of NeuN immunoreactive neurons was taken at the magnification of 20X using light microscope (BX53) (Olympus, Tokyo, Japan) equipped with digital camera (DP72) (Olympus). NeuN immunoreactive neurons were counted using image capture software (cellSens Standard) (Olympus). The total number of NeuN immunoreactive neurons was counted in 250 $\mu \mathrm{m}^{2}$ at the middle in the CA1 region, and the mean number was calculated using NIH Image (1.59 software) (National Institutes of Health, Bethesda, MD, USA).

To quantitatively analyze changes in the immunoreactivities of glial fibrillary acidic protein (GFAP), and IgG in the CA1 region, immunostained sections were observed in the same way described above. To evaluate changes in the immunoreactivities, in accordance with previous studies [12,25], all immunoreactive structures were converted into 8-bit grey scale images with a range of 0-255 (from black to white). Using Image J software (version 1.46) (National Institutes of Health), images were assessed for grey scale intensity, and average immunoreactive intensity in each immunoreactive staining was calculated within the area of interest. The immunoreactive intensity was relatively presented as relative optical density (ROD): the optical density of the Shamvehicle group was considered as $100 \%$.

\section{Histofluorescence using Fluoro-Jade B (F-J B)}

F-J B is used as a marker of cellular degeneration. F-J B histofluorescence was carried out in order to examine neuronal damage/death following TFI. As described previously $[12,26]$, in brief, the sections were incubated in $0.06 \%$ potassium permanganate $\left(\mathrm{KMnO}_{4}\right)$ (Sigma-Aldrich Co.) for $20 \mathrm{~min}$ on an orbital shaker. After washing for two minutes, these sections were reacted in 0.0004\% F-J B (Histochem, Jefferson, AR, USA) for $30 \mathrm{~min}$. Thereafter, the brain sections were washed three times for two minutes and placed on a slide warmer until they were fully dried. The slides were cleared using xylene for one minute and coverslipped with dibutylphthalate polystyrene xylene (Sigma-Aldrich Co.).

The count of F-J B positive cells (neurons) was performed to evaluate TFI-induced neuronal death in the CA1 region. In short, according to published methods [4,24], the image of F-J B positive cells was taken at the magnification of 20X using epifluorescent microscope (Carl Zeiss, Oberkochen, Germany), equipped with a digital camera (DP72) (Olympus), at 450-490 nm of wavelength. F-J B positive cells were taken using image capture software (cellSens Standard) (Olympus). The total number of F-J B positive cells was counted in the same way described above (count of NeuN immunoreactive neurons).

\section{Double immunofluorescence staining}

To distinguish astrocyte endfeet and blood vessels, double immunofluorescence staining was conducted according to a published protocol [12]. In short, the sections were reacted in mouse anti-GFAP (dilute rate, 1:800) (Chemicon, Temecula, CA, USA), mouse anti-NeuN (dilute rate, 1:800) (Chemicon), rabbit antiglucose transporter 1 (GLUT-1; a marker for endothelial cells) (dilute rate 1:1,000) (Chemicon), and rabbit anti-IgG (dilute rate, 1:800) (Bioss antibodies, Atlanta, GA, USA) were used as primary antibodies. Thereafter, the sections were immunoreacted with secondary antibodies combined Alexa Fluor 488-conjugated donkey anti-mouse IgG (dilute rate, 1:500) (Invitrogen, Waltham, MA, USA) and Alexa Fluor 546-conjugated goat anti-rabbit IgG (dilute rate 1:500) (Invitrogen). After washing, the sections were mounted onto the slide glasses and dehydrated using a dry oven (WiseVen WOC High Clean Air Oven) (Daihan Scientific Co., Ltd., Wonju, Korea) for eight hours. Finally, the sections were coverslipped with DPX (Sigma-Aldrich Co.).

The immunoreaction of GFAP/GLUT-1 was observed using confocal MS (LSM510 META NLO) (Carl Zeiss), which was in the Korea Basic Science Institute Chuncheon Center (Chuncheon, Korea).

\section{Statistical analysis}

In this study, we used SPSS software (version 15.0) (SPSS Inc., Chicago, IL, USA) to perform statistical analyses. Kolmogorov and Smirnov test was used to test normal distributions, and Bartlett test was used to test identical standard error of the means (SEMs). All our data passed the normality test. The statistical significances of the mean among the experimental groups were determined by two-way analysis of variance (for the numbers of NeuN-immunoreactive neurons and F-J B positive cells, RODs of GFAP and IgG-immunoreactive structures, and latency times in PAT) and repeated measures analysis of variance (for the numbers of errors in 8-ARMT) followed by post-hoc Tukey test for all pairwise multiple comparisons. Statistical significance was considered to be at a value of $\mathrm{p}<0.05$. 


\section{RESULTS}

\section{Effect of STP against TFI-induced cognitive deficit}

In this study, as shown in the Fig. 2, STP treatment after TFI significantly attenuated TFI-induced cognitive impairments. In PAT, the latency time one day before TFI was not significantly different between the Sham-vehicle and all Sham-STP groups, and, five days after TFI, the latency time in all Sham groups was not different from that shown one day before TFI (Fig. 2A). In the TFI-vehicle, TFI-10 mg/kg STP and TFI-15 mg/kg STP groups, the latency time five days after TFI was significantly lower than that in the Sham-vehicle and Sham-STP groups (Fig. 2A). However, five days after TFI, the latency time in the TFI- $20 \mathrm{mg} / \mathrm{kg}$ STPgroup was similar to that found in the Sham-vehicle group (Fig. 2A).

In 8-ARMT, the number of the errors was gradually decreased in all groups with time before TFI, and the decrease pattern was not difference between all groups (Fig. 2B). One day after TFI, the numbers of the errors were significantly increased in the TFIvehicle and all TFI-STP groups when compared with the Shamvehicle and all Sham-STP groups (Fig. 2B). From two days to five days after TFI, the numbers of the errors in the TFI-vehicle, TFI$10 \mathrm{mg} / \mathrm{kg}$ STP and $15 \mathrm{mg} / \mathrm{kg}$ STP groups were not significantly decreased with time after TFI (Fig. 2B). However, the numbers of the errors in the TFI- $20 \mathrm{mg} / \mathrm{kg}$ STP group began to decrease from two days after TFI and were significantly decreased with time, showing that the numbers five days after TFI were similar to those shown in the Sham groups (Fig. 2B).

\section{Effect of STP against TFI-induced neuronal death}

In this experiment, we found that STP treatment after TFI protected neurons in the CA1 region from TFI injury (Fig. 3). In the Sham-vehicle group, neurons immunostained with $\mathrm{NeuN}\left(\mathrm{NeuN}^{+}\right.$ neurons), as intact neurons, were located in the stratum pyramidale, and cells positive to F-J B (F-J B ${ }^{+}$cells), as damaged (dead) cells, were not found (Fig. 3A, 3a, 3I, 3i). On the other hand, in the TFI-vehicle group, very few $\mathrm{NeuN}^{+}$neurons and many F-J $\mathrm{B}^{+}$cells were shown in the stratum pyramidale five days after TFI (Fig. 3B, 3b, 3I, 3i).

In all Sham-STP groups, no significant differences in the numbers of NeuN ${ }^{+}$and F-J B ${ }^{+}$cells were shown when compared with those found in the Sham-vehicle group (Fig. 3C, 3c, 3E, 3e, 3G, 3g, 3I, 3i). In the TFI-10 mg/kg STP and TFI-15 mg/kg STP groups, the distribution of $\mathrm{NeuN}^{+}$and F-J B ${ }^{+}$cells was very similar to the TFI-vehicle group (Fig. 3D, 3d, 3F, 3f, 3I and 3i). In contrast, in the TFI-20 mg/kg STP group, many $\mathrm{NeuN}^{+}$and very few F-J $\mathrm{B}^{+}$cells were detected in the stratum pyramidale (Fig. 3H, 3h, 3I, 3i): this finding means that therapeutic treatment with $20 \mathrm{mg} / \mathrm{kg}$ STP after TFI in gerbils very effectively protected hippocampal neurons from ischemia-reperfusion injury.

Based on these results, we described the following results obtained from the TFI- $20 \mathrm{mg} / \mathrm{kg}$ STP group.
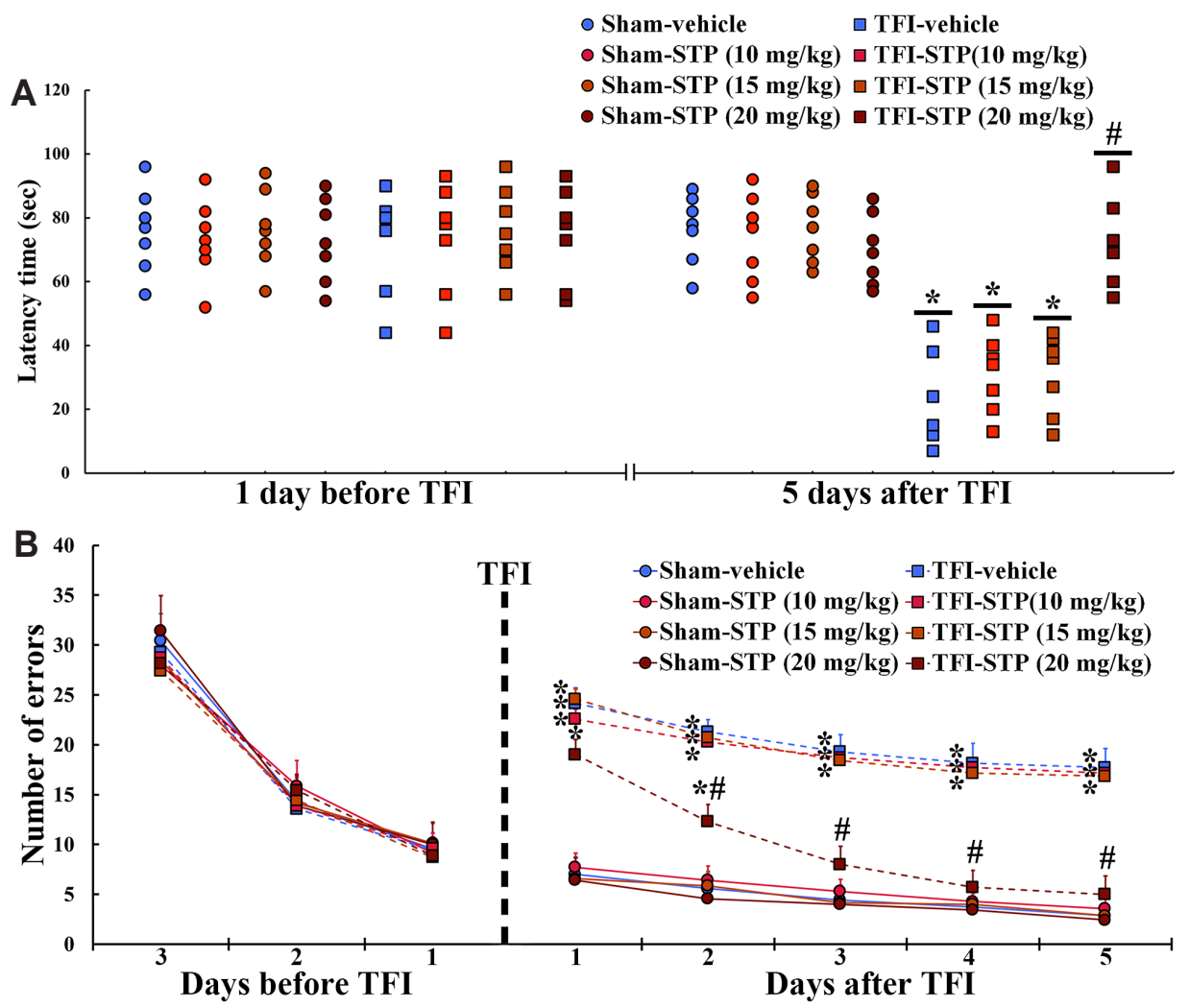

Fig. 2. Latency time in PAT (A) and mean numbers of errors in 8-ARMT (B) in the Sham-vehicle, Sham-STP (10, 15 , and $20 \mathrm{mg} / \mathrm{kg}$ ), TFI-vehicle and TFI-STP $(10,15$, and $20 \mathrm{mg} / \mathrm{kg})$ groups before and after TFI. The substantial trials for PAT are conducted one day before and five days after TFI and gerbils are undergone pre-training trial at 20 min before each substantial trial. In the TFI-20 mg/kg STP group, a significantly delayed latency time in PAT is recorded compared with that in the TFI-vehicle, TFI-10 mg/kg STP and TFI- 15 mg/kg STP groups. 8-ARMT is daily performed at three to one day before TFI, and one to five days after TFI. In the TFI- $20 \mathrm{mg} / \mathrm{kg}$ STP group, the numbers of the errors in 8-ARMT are significantly decreased from two days after TFI. The bars indicate the means \pm SEM $(n=7$, respectively; ${ }^{*} \mathrm{p}<0.05$ vs. Sham-vehicle or Sham-STP group; ${ }^{*} p<0.05$ vs. TFI-vehicle group). 8-ARMT, 8-arm radial maze test; PAT, passive avoidance test; STP, stiripentol; TFI, transient forebrain ischemia. 

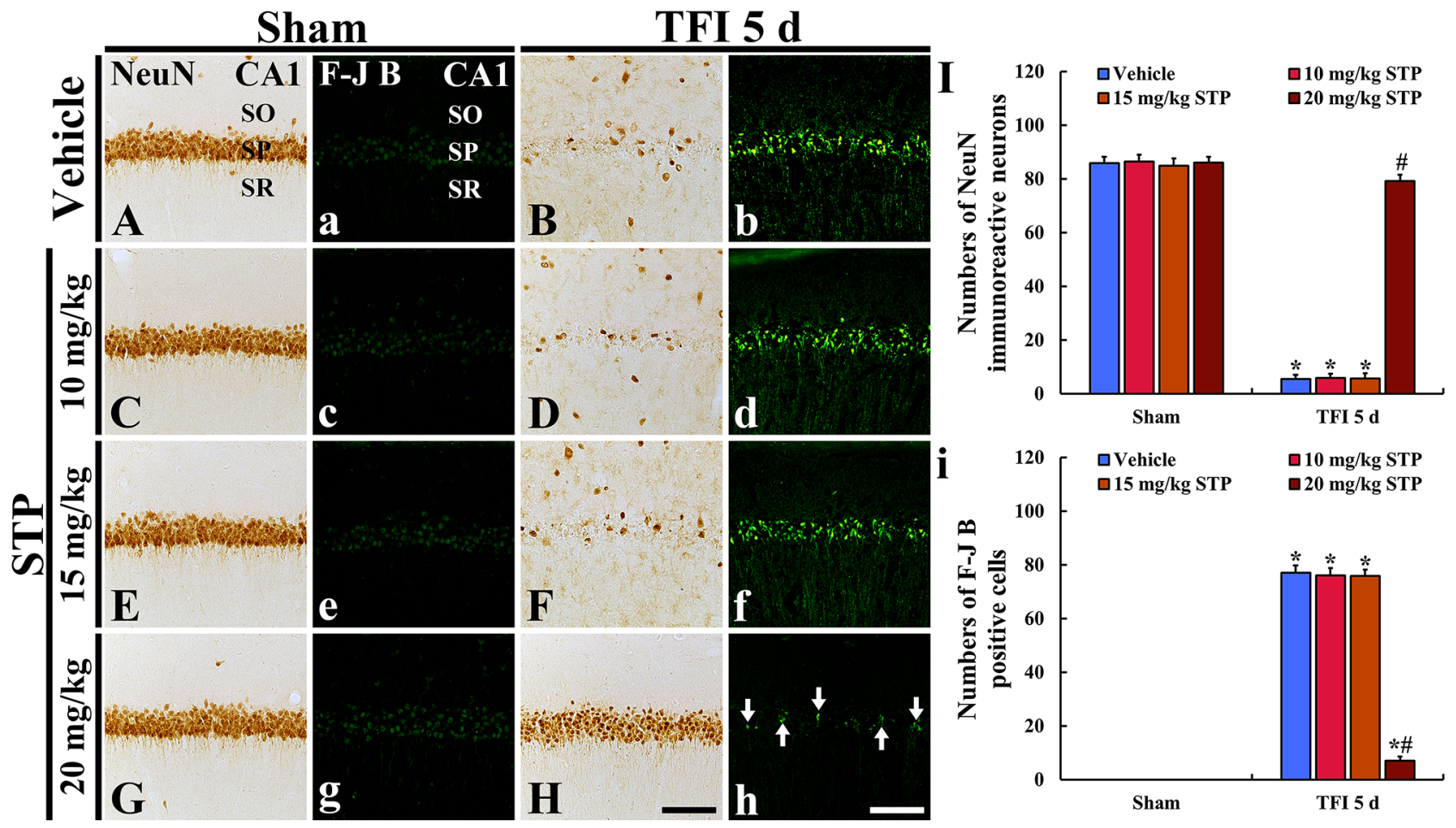

Fig. 3. (A-H and a-h) NeuN immunohistochemistry (A-H) and F-J B histofluorescence (a-h) in the CA1 region of the Sham-vehicle (A and a), TFI-vehicle (B and b), Sham-STP (10, 15, and $20 \mathrm{mg} / \mathrm{kg})(C, \mathrm{c}, \mathrm{E}, \mathrm{e}, \mathrm{G}$, and g) and TFI-STP (10, 15, and $20 \mathrm{mg} / \mathrm{kg})(\mathrm{D}, \mathrm{d}, \mathrm{F}, \mathrm{f}, \mathrm{H}$, and h) groups five days after TFI. In the TFI-vehicle group, NeuN ${ }^{+}$cells are rarely found, and F-J $\mathrm{B}^{+}$cells are abundant in the stratum pyramidale (SP). However, in the TFI-20 mg/kg STP group, NeuN ${ }^{+}$cells are abundant and F-J B ${ }^{+}$cells are very few (arrows) in the SP. Scale bars $=100 \mu \mathrm{m}$. (I and i) The mean numbers of NeuN $^{+}$cells (I) and F-J $B^{+}$cells (i). The bars indicate the means \pm SEM $\left(n=5\right.$ or 7 , respectively; ${ }^{*} p<0.05$ vs. Sham-vehicle or Sham-STP group; ${ }^{*} p<0.05$ vs. TFI-vehicle group). NeuN, neuronal nuclei; F-J B, Fluoro-Jade B; CA1, cornu ammonis 1; TFI, transient forebrain ischemia; STP, stiripentol; SO, stratum oriens; SR, stratum radiatum.

\section{Effect of STP against TFI-induced astrocyte damage}

We found, in this study, that STP treatment after TFI significantly attenuated TFI-induced astrocyte damage in the CA1 region (Fig. 4). In the Sham-vehicle group, cells immunostained with GFAP, as intact GFAP ${ }^{+}$astrocytes, had thin processes and small cytoplasm, and most of them were distributed in stratum oriens and radiatum (Fig. 4Aa). In the TFI-vehicle group, GFAP immunoreactivity in the astrocytes was increased with time after TFI, showing that their cytoplasm and processes became gradually hypertrophied and thickened, respectively (Fig. 4Ab-Ad and 4B).

No significant difference was shown in GFAP immunoreactivity between the Sham- $20 \mathrm{mg} / \mathrm{kg}$ STP and the Sham-vehicle groups (Fig. 4Ae, 4B). In the TFI-20 mg/kg STP group, the hypertrophy of the $\mathrm{GFAP}^{+}$astrocytes was significantly attenuated after TFI when compared with those found in the Sham-vehicle group (Fig. $4 \mathrm{Af}-\mathrm{Ah}$ and $4 \mathrm{~B})$.

To examine the changes of the ends of astrocyte processes, as astrocyte endfeet (AEF), we performed double immunofluorescence for GFAP and GLUT-1 in the CA1 region after TFI. In all Sham groups, $\mathrm{GFAP}^{+} \mathrm{AEF}$ were found around GLUT- ${ }^{+}$ endothelial cells (Fig. 5A, E). In the TFI-vehicle group, the AFE disappeared gradually after TFI, showing that, five days after TFI, most of $\mathrm{GFAP}^{+} \mathrm{AFE}$ were not contact with small blood vessels (Fig. 5B-D). On the other hand, the damage of $\mathrm{GFAP}^{+} \mathrm{AEF}$ was significantly attenuated in the TFI- $20 \mathrm{mg} / \mathrm{kg}$ STP-group (Fig. 5F$\mathrm{H})$.

\section{Effect of STP against TFI-induced lgG leakage}

As shown in the Fig. 6, in this study, we found that STP treatment after TFI significantly attenuated TFI-induced IgG leakage in the CA1 region. In the Sham-vehicle group, IgG immunoreactivity was shown inside blood vessels, not in CA1 parenchyma (Fig. 6Aa). In the TFI-vehicle group, IgG immunoreactivity was shown in cells six hours after TFI, apparently increased in the parenchyma and cells two days after TFI, and very high five days after TFI (Fig. 6Ab-Ad and 6B).

In the Sham- $20 \mathrm{mg} / \mathrm{kg}$ STP group, IgG immunoreactivity was not different from the Sham-vehicle group (Fig. 6Ae). In the TFI$20 \mathrm{mg} / \mathrm{kg}$ STP group, IgG immunoreactivity was significantly lower than that in the TFI-vehicle group, although the distribution pattern of $\operatorname{IgG}^{+}$structures was similar to that shown in the TFI-vehicle group (Fig. 6Af-Ah and 6B). 


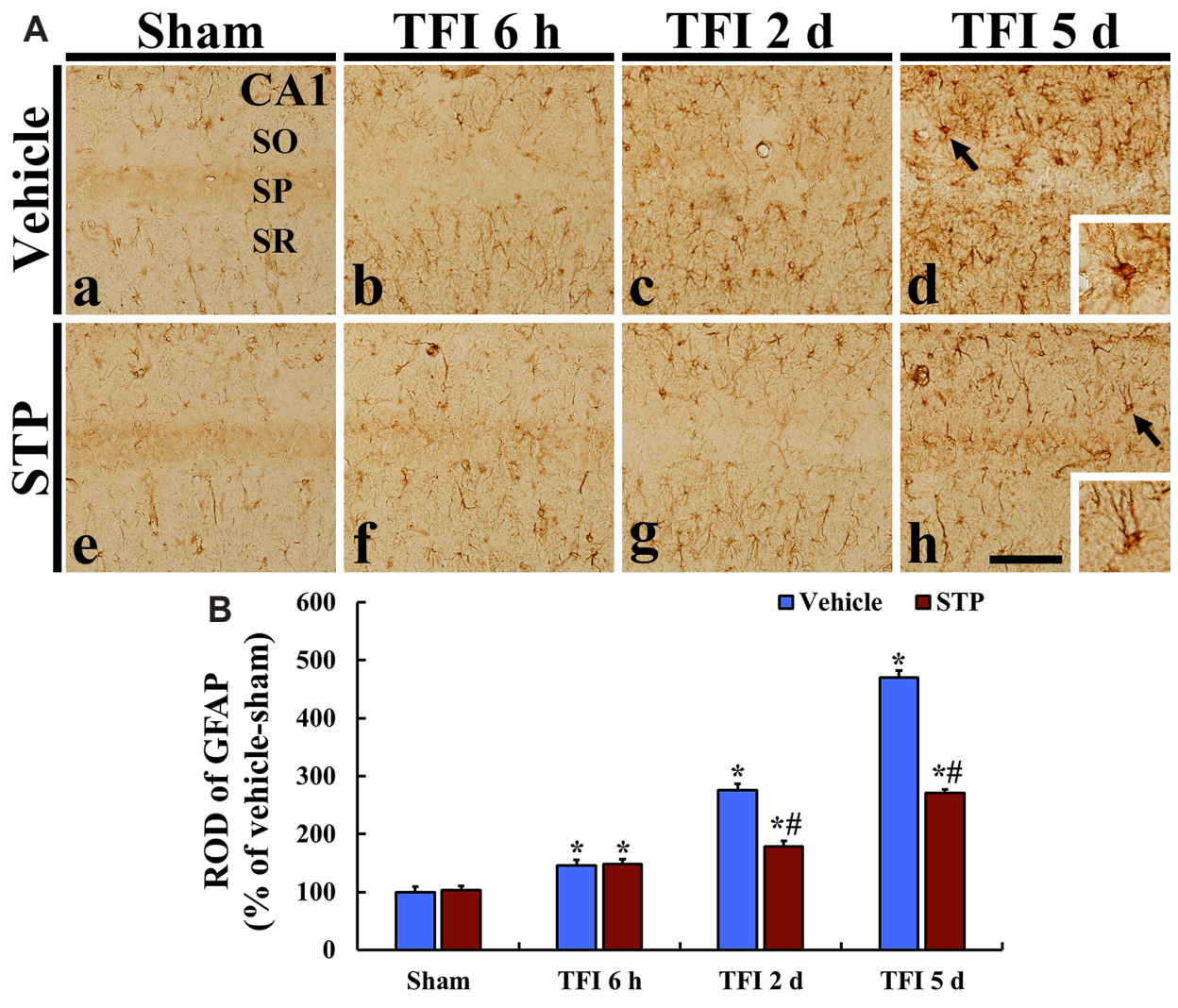

Fig. 4. (A) GFAP immunohistochemistry in the CA1 region of the Shamvehicle (a), TFI-vehicle (b-d), Sham-20 $\mathrm{mg} / \mathrm{kg}$ STP (e) and TFI-20 mg/kg STP (f-h) groups six hours (b and f), two days ( $c$ and $g$ ) and five days ( $d$ and $h$ ) after TFI. In the TFI-vehicle group, GFAP ${ }^{+}$ astrocytes become gradually hypertrophied after TFI, showing that the ends of the astrocytes are blunt (arrow) five days after TFI. In contrast, the hypertrophy of $\mathrm{GFAP}^{+}$astrocytes is apparently attenuated in the TFI-20 mg/kg STP group. Scale bars $=100 \mu \mathrm{m}$. (B) ROD of GFAP immunoreactive structures. The bars indicate the means \pm SEM $(n=5$ or 7 , respectively; ${ }^{*} p<0.05 v$ vs. Sham-vehicle or Sham-STP group; ${ }^{*} p<0.05$ vs. TFIvehicle group). GFAP, glial fibrillary acidic protein; CA1, cornu ammonis 1; TFI, transient forebrain ischemia; STP, stiripentol; $\mathrm{ROD}$, relative optical density; $\mathrm{SO}$, stratum oriens; SP, stratum pyramidale; SR, stratum radiatum.

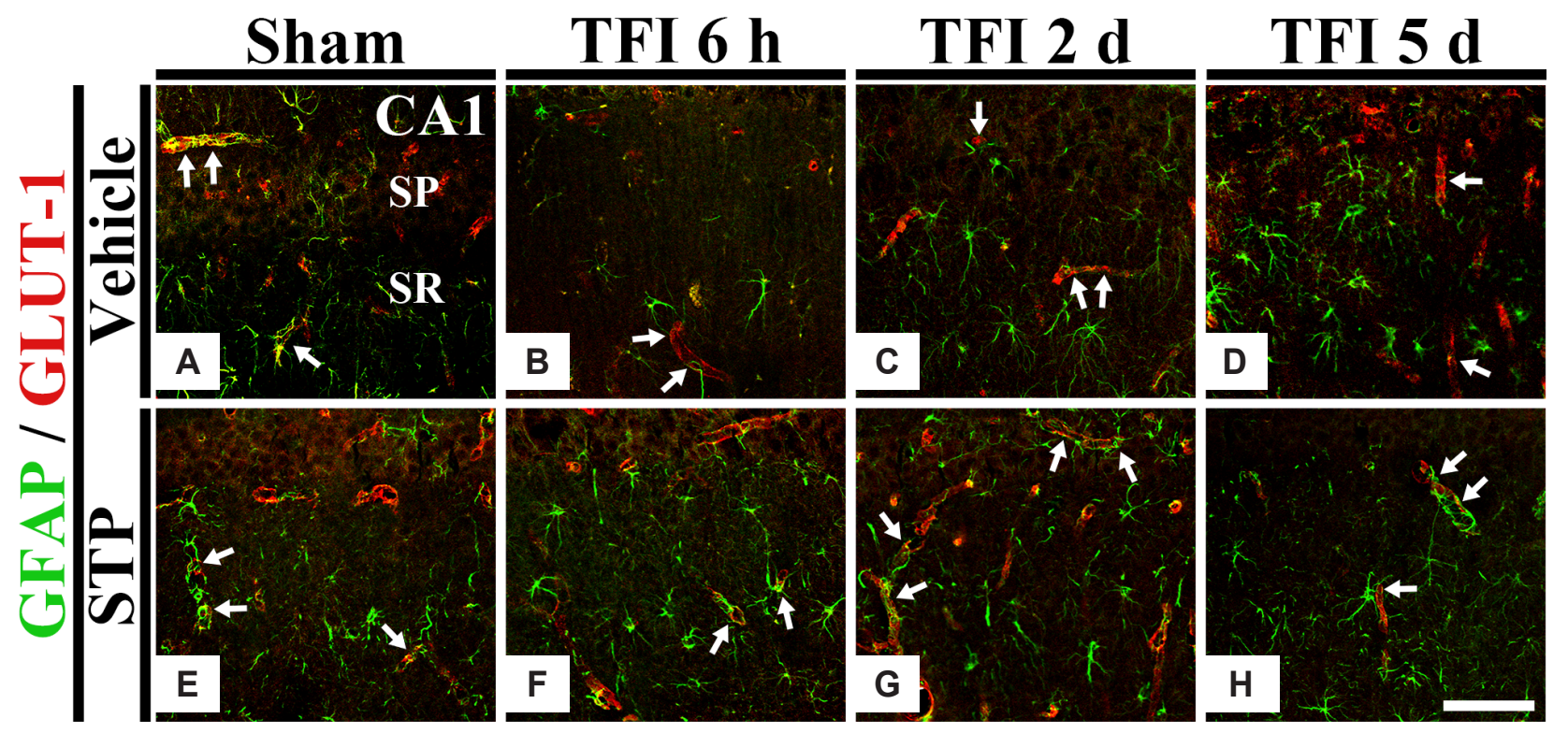

Fig. 5. Double immunohistofluorescence for GFAP (green) and GLUT-1 (red) in the CA1 region of the Sham-vehicle (A), TFI-vehicle (B-D), Sham-20 mg/kg STP (E) and TFI-20 mg/kg STP (F-H) groups six hours (B and F), two days (C and G) and five days (D and H) after TFI. GFAP AEF (arrows in A) are contact with GLUT-1 ${ }^{+}$endothelial cells in the Sham-vehicle group; however, in the TFI-vehicle group, AEF disappear gradually (arrows in B-D). In contrast, GFAP ${ }^{+}$AEF (arrows in F-H) are well co-localized with GLUT- $1^{+}$endothelial cells in the TFI- $20 \mathrm{mg} / \mathrm{kg}$ STP group. Scale bars $=50 \mu \mathrm{m}$. GFAP, glial fibrillary acidic protein; GLUT-1, glucose transporter 1; CA1, cornu ammonis 1; TFI, transient forebrain ischemia; STP, stiripentol; AEF, astrocyte endfeet; SP, stratum pyramidale; SR, stratum radiatum.

\section{DISCUSSION}

In this study, to investigate the therapeutic effect of STP against ischemic injury induced by TFI and its possible mechanism, cognitive impairment, neuronal death, astrocyte damage, IgG leakage and microgliosis were examined in the CA1 region of gerbil 


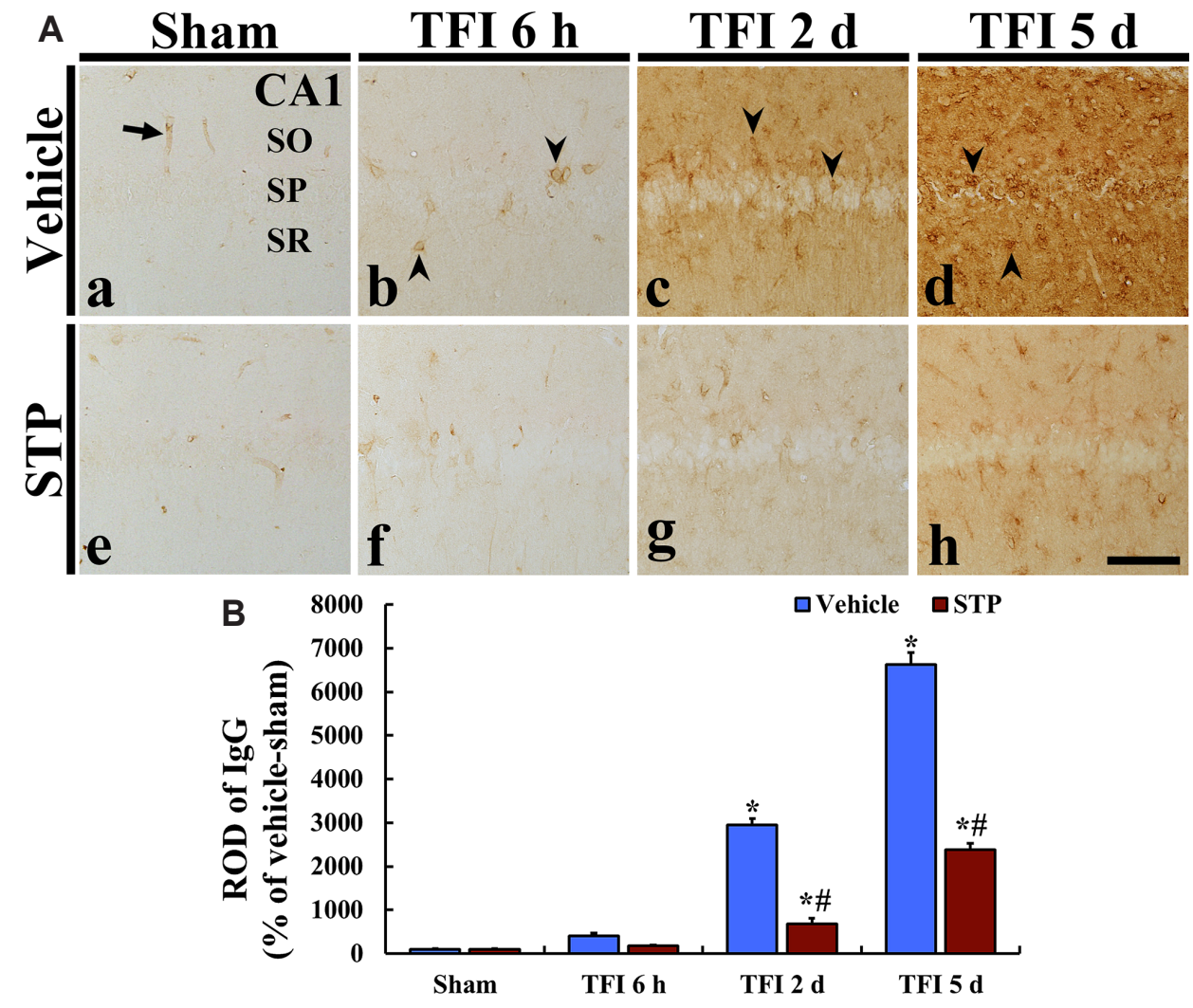

Fig. 6. (A) IgG immunohistochemistry in the CA1 region of the Shamvehicle (a), TFI-vehicle (b-d), Sham$20 \mathrm{mg} / \mathrm{kg}$ STP (e) and TFI-20 mg/kg STP (f-h) groups six hours (b and f), two days ( $c$ and $g$ ) and five days (d and $\mathbf{h}$ ) after TFI. In the Sham-vehiclegroup, IgG immunoreactivity is shown only within blood vessels (arrow). In the TFI-vehicle-group, IgG immunoreactivity is increased in cellular components (arrowheads) and parenchyma from six hours after TFI. In contrast, IgG immunoreactivity in the TFI- $20 \mathrm{mg} / \mathrm{kg}$ STP group is apparently decreased. Scale bars = $100 \mu \mathrm{m}$. (B) ROD of IgG immunoreactive structures. The bars indicate the means \pm SEM ( $n=5$ or 7 , respectively; ${ }^{*} p<0.05$ vs. Sham-vehicle or Sham-STP group; ${ }^{\#} \mathrm{p}<0.05$ vs. TFI-vehicle group). IgG, immunoglobulin $\mathrm{G}$; CA1, cornu ammonis 1; TFI, transient forebrain ischemia; STP, stiripentol; ROD, relative optical density; $\mathrm{SO}$, stratum oriens; SP, stratum pyramidale; $S R$, stratum radiatum. hippocampus known to be vulnerable to transient ischemia, following 5-min TFI.

It is well known that TFI-induced hippocampal neuronal damage is closely associated with deficits in learning and memory and that impaired cognitive performance can occur before the onset of TFI-induced neuronal loss (death) in the hippocampus [1012,27]. PAT and 8-ARMT have been widely used to investigate alterations of cognitive function following ischemic injury in the hippocampus induced by ischemia-reperfusion [11,12,22,28]. Therefore, in the present study, to examine whether STP treatment after TFI in gerbils affected TFI-induced change in cognitive function, we performed PAT and 8-ARMT. Result of PAT revealed that the latency time in the TFI- $20 \mathrm{mg} / \mathrm{kg}$ STP group was very similar to that in the Sham-vehicle group five days after TFI. Result of 8-ARMT showed that the number of errors in the TFI$20 \mathrm{mg} / \mathrm{kg}$ STP group was not significantly different from that in the Sham-vehicle group. These results indicate that $20 \mathrm{mg} / \mathrm{kg}$ STP (not 10 and 15 STP) treatment after TFI can improve TFI-induced cognitive impairment.

Some previous studies have reported the neuroprotective effect of STP in experimental animal models of neurological disorders. Auvin et al. [16] reported that STP reduced neuronal injury in the hippocampus of a rat model of status epilepticus induced by lithium-pilocarpine. In particular, Verleye et al. [29] used two in vitro models of brain injury associated with seizure through neuronalastroglial cultures from rat cerebral cortex exposed to high level of glutamate ( $40 \mu \mathrm{M}$ for $20 \mathrm{~min}$ ) or to oxygen-glucose deprivation
(OGD) and found that STP had neuroprotective effects when it was used for treatment before, but not after, exposure to GOD, whereas neuroprotective effects by STP was found when it was treated both before and after exposure to a high level of glutamate. In addition, Chatterjee et al. [30] recently reported that STP had a neuroprotective effect against neurotoxicity in PC12 cells induced by treatment with anti-nerve growth factor and that STP treatment reduced neurotoxicity in rat primary cortical neurons induced by $A \beta 42$ implicated in Alzheimer's disease. In the present study, we examined neuroprotective effects of post-treatment with STP against TFI-induced ischemic damage in gerbil hippocampal CA1 region using NeuN immunohistochemical staining and F-J B histofluorescence, and found that post-treatment with STP at $20 \mathrm{mg} / \mathrm{kg}$, but not at 10 or $15 \mathrm{mg} / \mathrm{kg}$, protected against TFI-induced delayed neuronal death in the hippocampal CA1 region. Based on our results and previous findings, to the best of our knowledge, this is the first study showing that STP had a neuroprotective effect against ischemia-reperfusion injury using a gerbil model of TFI. This result is correlated with results of the two behavioral tests (PAT and 8-ARMT).

Astrocytes (astroglial cells) are the most common glial cells located in the central nervous system (CNS; brain and spinal cord). They perform diverse functions including maintenance of extracellular ion balance, support of endothelial cells for the constitution of $\mathrm{BBB}$, nutrient provision to nervous tissues, and participation in repair of CNS injuries [31-33]. It has been reported that astrocytes play significant roles in both healthy and 
injured brains including ischemic stroke [34-37]. Functional and morphological alterations in astrocytes have been reported in brains with pathological conditions (the process is termed "astrogliosis") $[2,38,39]$. It is known that astrogliosis is characterized by proliferation and hypertrophy of astrocytes with increased GFAP expression in astrocytes $[2,40]$. However, Sofroniew and Vinters [41] have reported that an increase of cells positive to GFAP does not result from new astrocyte generation, but from an increase in GFAP synthesis and filament condensation in pre-existing astrocytes. Based on this finding, we have recently reported that GFAP positive cells (astrocytes) with strong GFAP immunoreactivity in gerbil striatum are swollen in cell bodies and destroyed (short and thickened) in their processes at five days after TFI [39]. In the current study, GFAP immunoreactivity was significantly increased in astrocytes of the TFI-vehicle group. Their cytoplasm and processes became gradually hypertrophied and thickened. Furthermore, when we performed double immunofluorescence for GFAP and GLUT-1, AFE (ends of astrocyte processes) disappeared seriously at five days after TFI. Most of the GFAP positive AFE were not having contacts with small blood vessels. However, in the TFI-20 $\mathrm{mg} / \mathrm{kg}$ STP group, the hypertrophy of GFAP positive astrocytes was significantly attenuated after TFI. Furthermore, the damage of AEF was significantly attenuated. For reference on AEF, Abbott [42] reported that the AEF of astrocytes envelops almost all (> 99\%) endothelium in brains. In addition, Daneman and Prat [32] have reported that astrocytes provide cellular linkages between neurons and small blood vessels by AFE which is a part of the BBB [2]. Taken together, our results suggest that the damage of astrocytes (in particular, AFE) in the hippocampus after TFI is involved in TFI-induced neuronal loss (death), which is related to cognitive deficit, and that this damage can be attenuated or protected by $20 \mathrm{mg} / \mathrm{k}$ STP.

Finally, we investigated whether post-treatment with $20 \mathrm{mg} / \mathrm{kg}$ STP displayed a protective effect against TFI-induced BBB leakage. A healthy condition in the CNS is attained by BBB through preserving the balance between water and ion and supplying nutrients [31,32]. In addition, it is generally accepted that BBB integrity should be maintained to protect brain following an ischemic injury. Thus, maintenance of the BBB integrity is considered as one of potential therapeutic targets for treating an ischemic stroke [43-45]. In normal conditions, BBB as a selectively permeable barrier allows only specific substances from blood to enter brain parenchyma and participate in maintaining homeostasis in the CNS. However, disruption of BBB integrity, BBB leakage and marked extravasation can occur after an ischemic injury $[10,12,46,47]$. In many experiments, extravascular IgG has been used to examine the increase of BBB permeability $[10,12,47]$. Therefore, we performed IgG immunohistochemistry and found that IgG immunoreactivity was markedly increased in the CA1 region after TFI. We then we investigated the protective effect of STP against TFI-induced BBB leakage. We found that STP treatment significantly decreased IgG immunoreactivity in the isch- emic CA1 region. This finding indicates that STP treatment after TFI can protect the BBB integrity in ischemic brains.

In conclusion, this study showed that post-treatment with 20 $\mathrm{mg} / \mathrm{kg}$ STP ameliorated TFI-induced cognitive impairment and protected against TFI-induced neuronal death in the hippocampal CA1 region. In addition, STP treatment markedly attenuated IgG leakage in the ischemic CA1 region. Results of this study suggest that STP can be developed as a therapeutic drug for treating ischemia-reperfusion injury or ischemic stroke.

\section{FUNDING}

This work was supported by Basic Science Research Program through the National Research Foundation of Korea (NRF) funded by the Ministry of Education (NRF-2016R1D1A1B01011790, NRF- 2020R1I1A3068251 and NRF- 2021R1F1A1055802).

\section{ACKNOWLEDGEMENTS}

The authors would like to appreciate Ms. Hyun Sook Kim and Mr. Seung Uk Lee for their technical help in this study.

\section{CONFLICTS OF INTEREST}

The authors declare no conflicts of interest.

\section{REFERENCES}

1. Kirino T, Sano K. Selective vulnerability in the gerbil hippocampus following transient ischemia. Acta Neuropathol. 1984;62:201-208.

2. Lee TK, Kim H, Song M, Lee JC, Park JH, Ahn JH, Yang GE, Kim $\mathrm{H}$, Ohk TG, Shin MC, Cho JH, Won MH. Time-course pattern of neuronal loss and gliosis in gerbil hippocampi following mild, severe, or lethal transient global cerebral ischemia. Neural Regen Res. 2019;14:1394-1403.

3. Kirino T. Delayed neuronal death in the gerbil hippocampus following ischemia. Brain Res. 1982;239:57-69.

4. Lee TK, Kang IJ, Kim B, Sim HJ, Kim DW, Ahn JH, Lee JC, Ryoo S, Shin MC, Cho JH, Kim YM, Park JH, Choi SY, Won MH. Experimental pretreatment with chlorogenic acid prevents transient ischemia-induced cognitive decline and neuronal damage in the hippocampus through anti-oxidative and anti-inflammatory effects. Molecules. 2020;25:3578.

5. Park JH, Kim YH, Ahn JH, Choi SY, Hong S, Kim SK, Kang IJ, Kim YM, Lee TK, Won MH, Lee CH. Atomoxetine protects against NMDA receptor-mediated hippocampal neuronal death following transient global cerebral ischemia. Curr Neurovasc Res. 2017;14:158168.

6. Puyal J, Ginet V, Clarke PG. Multiple interacting cell death mechanisms in the mediation of excitotoxicity and ischemic brain damage: 
a challenge for neuroprotection. Prog Neurobiol. 2013;105:24-48.

7. Farhadi Moghadam B, Fereidoni M. Neuroprotective effect of menaquinone-4 (MK-4) on transient global cerebral ischemia/reperfusion injury in rat. PLoS One. 2020;15:e0229769.

8. Victoria ECG, Toscano ECB, Oliveira FMS, de Carvalho BA, Caliari MV, Teixeira AL, de Miranda AS, Rachid MA. Up-regulation of brain cytokines and metalloproteinases 1 and 2 contributes to neurological deficit and brain damage in transient ischemic stroke. Microvasc Res. 2020;129:103973.

9. Ju F, Ran Y, Zhu L, Cheng X, Gao H, Xi X, Yang Z, Zhang S. Increased BBB permeability enhances activation of microglia and exacerbates loss of dendritic spines after transient global cerebral ischemia. Front Cell Neurosci. 2018;12:236.

10. Kho AR, Choi BY, Lee SH, Hong DK, Lee SH, Jeong JH, Park KH, Song HK, Choi HC, Suh SW. Effects of protocatechuic acid (PCA) on global cerebral ischemia-induced hippocampal neuronal death. Int J Mol Sci. 2018;19:1420.

11. Kondo T, Yoshida S, Nagai H, Takeshita A, Mino M, Morioka H, Nakajima T, Kusakabe KT, Okada T. Transient forebrain ischemia induces impairment in cognitive performance prior to extensive neuronal cell death in Mongolian gerbil (Meriones unguiculatus). $J$ Vet Sci. 2018;19:505-511.

12. Lee TK, Kang IJ, Sim H, Lee JC, Ahn JH, Kim DW, Park JH, Lee $\mathrm{CH}$, Kim JD, Won MH, Choi SY. Therapeutic effects of decursin and Angelica gigas Nakai root extract in gerbil brain after transient ischemia via protecting BBB leakage and astrocyte endfeet damage. Molecules. 2021;26:2161.

13. Inoue Y, Ohtsuka Y; STP-1 Study Group. Effectiveness of add-on stiripentol to clobazam and valproate in Japanese patients with Dravet syndrome: additional supportive evidence. Epilepsy Res. 2014;108:725-731.

14. Fisher JL. The effects of stiripentol on GABA(A) receptors. Epilepsia. 2011;52 Suppl 2:76-78.

15. Nickels KC, Wirrell EC. Stiripentol in the management of epilepsy. CNS Drugs. 2017;31:405-416.

16. Auvin S, Lecointe C, Dupuis N, Desnous B, Lebon S, Gressens P, Dournaud P. Stiripentol exhibits higher anticonvulsant properties in the immature than in the mature rat brain. Epilepsia. 2013;54:20822090.

17. Shen DD, Levy RH, Moor MJ, Savitch JL. Efficacy of stiripentol in the intravenous pentylenetetrazol infusion seizure model in the rat. Epilepsy Res. 1990;7:40-48.

18. Trojnar MK, Wojtal K, Trojnar MP, Czuczwar SJ. Stiripentol. A novel antiepileptic drug. Pharmacol Rep. 2005;57:154-160.

19. Fujiwara A, Nakao K, Ueno T, Matsumura S, Ito S, Minami T. Stiripentol alleviates neuropathic pain in L5 spinal nerve-transected mice. J Anesth. 2020;34:373-381.

20. Jarrott DM, Domer FR. A gerbil model of cerebral ischemia suitable for drug evaluation. Stroke. 1980;11:203-209.

21. Bae EJ, Chen BH, Yan BC, Shin BN, Cho JH, Kim IH, Ahn JH, Lee JC, Tae HJ, Hong S, Kim DW, Cho JH, Lee YL, Won MH, Park JH. Delayed hippocampal neuronal death in young gerbil following transient global cerebral ischemia is related to higher and longerterm expression of p63 in the ischemic hippocampus. Neural Regen Res. 2015;10:944-950.

22. Chen BH, Park JH, Lee YL, Kang IJ, Kim DW, Hwang IK, Lee CH, Yan BC, Kim YM, Lee TK, Lee JC, Won MH, Ahn JH. Melatonin improves vascular cognitive impairment induced by ischemic stroke by remyelination via activation of ERK1/2 signaling and restoration of glutamatergic synapses in the gerbil hippocampus. Biomed Pharmacother. 2018;108:687-697.

23. Radtke-Schuller S, Schuller G, Angenstein F, Grosser OS, Goldschmidt J, Budinger E. Brain atlas of the Mongolian gerbil (Meriones unguiculatus) in CT/MRI-aided stereotaxic coordinates. Brain Struct Funct. 2016;221 Suppl 1:1-272.

24. Sharma SS, Dhar A, Kaundal RK. FeTPPS protects against global cerebral ischemic-reperfusion injury in gerbils. Pharmacol Res. 2007;55:335-342.

25. Meurer RT, Martins DT, Hilbig A, Ribeiro Mde C, Roehe AV, Barbosa-Coutinho LM, Fernandes Mda C. Immunohistochemical expression of markers Ki-67, neun, synaptophysin, p53 and HER2 in medulloblastoma and its correlation with clinicopathological parameters. Arq Neuropsiquiatr. 2008;66(2B):385-390.

26. Schmued LC, Hopkins KJ. Fluoro-Jade B: a high affinity fluorescent marker for the localization of neuronal degeneration. Brain Res. 2000;874:123-130.

27. Block F, Schwarz M. Correlation between hippocampal neuronal damage and spatial learning deficit due to global ischemia. Pharmacol Biochem Behav. 1997;56:755-761.

28. Pegorini S, Braida D, Verzoni C, Guerini-Rocco C, Consalez GG, Croci L, Sala M. Capsaicin exhibits neuroprotective effects in a model of transient global cerebral ischemia in Mongolian gerbils. $\mathrm{Br}$ J Pharmacol. 2005;144:727-735.

29. Verleye M, Buttigieg D, Steinschneider R. Neuroprotective activity of stiripentol with a possible involvement of voltage-dependent calcium and sodium channels. J Neurosci Res. 2016;94:179-189.

30. Chatterjee T, Das G, Chatterjee BK, Dhar J, Ghosh S, Chakrabarti P. The role of isoaspartate in fibrillation and its prevention by ProteinL-isoaspartyl methyltransferase. Biochim Biophys Acta Gen Subj. 2020;1864:129500.

31. Horner PJ, Palmer TD. New roles for astrocytes: the nightlife of an 'astrocyte'. La vida loca! Trends Neurosci. 2003;26:597-603.

32. Daneman R, Prat A. The blood-brain barrier. Cold Spring Harb Perspect Biol. 2015;7:a020412.

33. Harukuni I, Bhardwaj A. Mechanisms of brain injury after global cerebral ischemia. Neurol Clin. 2006;24:1-21.

34. Anderson MF, Blomstrand F, Blomstrand C, Eriksson PS, Nilsson M. Astrocytes and stroke: networking for survival? Neurochem Res. 2003;28:293-305.

35. Bylicky MA, Mueller GP, Day RM. Mechanisms of endogenous neuroprotective effects of astrocytes in brain injury. Oxid Med Cell Longev. 2018;2018:6501031.

36. Liu Z, Chopp M. Astrocytes, therapeutic targets for neuroprotection and neurorestoration in ischemic stroke. Prog Neurobiol. 2016;144:103-120.

37. Park JH, Kim DW, Lee TK, Park CW, Park YE, Ahn JH, Lee HA, Won $\mathrm{MH}$, Lee $\mathrm{CH}$. Improved $\mathrm{HCN}$ channels in pyramidal neurons and their new expression levels in pericytes and astrocytes in the gerbil hippocampal CA1 subfield following transient ischemia. Int $J$ Mol Med. 2019;44:1801-1810.

38. Lee TK, Ahn JH, Park CW, Kim B, Park YE, Lee JC, Park JH, Yang GE, Shin MC, Cho JH, Kang IJ, Won MH. Pre-treatment with laminarin protects hippocampal CA1 pyramidal neurons and attenuates reactive gliosis following transient forebrain ischemia in gerbils. 
Mar Drugs. 2020;18:52.

39. Lee TK, Lee JC, Kim JD, Kim DW, Ahn JH, Park JH, Kim HI, Cho JH, Choi SY, Won MH, Kang IJ. Populus tomentiglandulosa extract is rich in polyphenols and protects neurons, astrocytes, and the blood-brain barrier in gerbil striatum following ischemia-reperfusion injury. Molecules. 2021;26:5430.

40. Kim H, Park JH, Shin MC, Cho JH, Lee TK, Kim H, Song M, Park CW, Park YE, Lee JC, Ryoo S, Kim YM, Kim DW, Hwang IK, Choi SY, Won MH, Ahn JH. Fate of astrocytes in the gerbil hippocampus after transient global cerebral ischemia. Int J Mol Sci. 2019;20:845.

41. Sofroniew MV, Vinters HV. Astrocytes: biology and pathology. Acta Neuropathol. 2010;119:7-35.

42. Abbott NJ. Astrocyte-endothelial interactions and blood-brain barrier permeability. J Anat. 2002;200:629-638.

43. Huang Y, Chen S, Luo Y, Han Z. Crosstalk between Inflammation and the BBB in Stroke. Curr Neuropharmacol. 2020;18:1227-1236.

44. Wang Z, Leng Y, Tsai LK, Leeds P, Chuang DM. Valproic acid at- tenuates blood-brain barrier disruption in a rat model of transient focal cerebral ischemia: the roles of HDAC and MMP-9 inhibition. $J$ Cereb Blood Flow Metab. 2011;31:52-57.

45. Zhang H, Park JH, Maharjan S, Park JA, Choi KS, Park H, Jeong Y, Ahn JH, Kim IH, Lee JC, Cho JH, Lee IK, Lee CH, Hwang IK, Kim YM, Suh YG, Won MH, Kwon YG. Sac-1004, a vascular leakage blocker, reduces cerebral ischemia-reperfusion injury by suppressing blood-brain barrier disruption and inflammation. J Neuroinflammation. 2017;14:122.

46. Lin CY, Chang C, Cheung WM, Lin MH, Chen JJ, Hsu CY, Chen $\mathrm{JH}$, Lin TN. Dynamic changes in vascular permeability, cerebral blood volume, vascular density, and size after transient focal cerebral ischemia in rats: evaluation with contrast-enhanced magnetic resonance imaging. J Cereb Blood Flow Metab. 2008;28:1491-1501.

47. Ma F, Sun P, Zhang X, Hamblin MH, Yin KJ. Endothelium-targeted deletion of the miR-15a/16-1 cluster ameliorates blood-brain barrier dysfunction in ischemic stroke. Sci Signal. 2020;13:eaay5686. 